\title{
Transabdominal Pre-Peritoneal Mesh for Inguinal Hernia Repair with External Fixation versus Mesh Stapling
}

\author{
Mohamed Abdelhamid*, Ahmed Mohamed Sadat, Ayman Refaat Abdelhaseeb, \\ Tamer Mohamed Nabil, Mohamed Salah Abdelbasset, Amro Mohamed Ali Bechet, \\ Hesham Ahmed Nafady, Kalid Ahmed Shawky \\ Faculty of Medicine, Bani Swif University, Bani Swif, Egypt \\ Email: *mohamedsalah_2000@hotmail.com
}

Received June 1, 2013; revised July 1, 2013; accepted July 10, 2013

Copyright (C) 2013 Mohamed Abdelhamid et al. This is an open access article distributed under the Creative Commons Attribution License, which permits unrestricted use, distribution, and reproduction in any medium, provided the original work is properly cited.

\begin{abstract}
Background: It is unknown whether stapling the mesh affects recurrence rate, incidence of neuralgia, and port-site hernia. We chose to fix it to the exterior reducing port size, cost and pain, at the same comparing this with traditional mesh stapling. Methods: We conducted a prospective trial for laparoscopic TAPP inguinal hernia repair on 120 patients in which we fixed the mesh to the anterior abdominal wall using either two prolene threads that passed to the exterior and tied in place or traditional mesh stapling. Results: The operative time is ranged from 35 to 70 minutes for external fixation, 30 to 60 minutes for mesh stapling, and 4 to 51 months for follow-up, and no recurrence occurred in both groups during the procedure. Two cases with post TAPP pain in mesh stapling patients are discussed with reduction of the cost and port size in external fixation patients. Conclusion: It is not necessary to secure the mesh during laparoscopic TAPP inguinal hernia repair from the interior and it is fixed only to the exterior allowing a reduction in size of the ports and considerable reduction in cost with elimination of TAPP associated post operative pain.
\end{abstract}

Keywords: Laparoscopic Transabdominal Preperitoneal Inguinal Hernia Repair; Mesh Prosthesis Fixation; Cost-Stapling

\section{Introduction}

3500 years ago, Egyptian physicians reported the management of hernia by conservative means including the snuggly fitting bandage for reduction and support. For 100 years, the Bassini-type repair for inguinal hernia was the standard method. The Lichtenstein "tension free" mesh repair replaced it on the grounds of much lower recurrence rates, $<5 \%$ vs. $\sim 15 \%$. However, all open procedures have significant long-term discomfort rates of up to $53 \%$. Laparoscopic repair has become a genuine option in the last 15 years and offers low recurrence $(<1 \%)$ and minimal long-term discomfort. However, it has not been widely taken up [1]. Laparoscopic inguinal hernia repair (LIHR) has comparable results to open hernia repair (OHR).

Many studies have shown that LIHR gives similar results in terms of recurrence as compared with OHR but with the added advantage of less chance of post operative pain, wound infection and early return to activity [2]. ${ }^{*}$ Corresponding author.
Following the laparoscopic revolution, laparoscopic hernia repair has become one of commoner laparoscopic operations. Several studies have demonstrated a definite advantage over open repair with regard to reduced postoperative pain [3-5] and earlier return to work and normal activities [6-8]. It is unknown at present what the best method is among mesh implantation, central incision, reconstructing the deep inguinal ring, or a non-incised mesh implant in laparoscopic hernia surgery [9]. No influence on postoperative complaints or complications could be demonstrated by different mesh fashioning and fixation alternatives [10]. Cost has been a major point of criticism against the laparoscopic approach, particularly in term of disposable items of which a stapling device is the most expensive [11]. That is why we tried to find out a way that can eliminate the use of expensive devices to reduce the cost without affecting the outcome.

\section{Patients}

This work was conducted at Al Hayat Hospital Jeddah, 
KSA, Bani Swif University Hospital and Bani Swif Health Insurance Hospital between September 2008 and August 2012. Included in the study were 120 patients with unilateral inguinal hernia, both direct and indirect. Both bilateral and recurrent hernia were excluded from the study. There were 42 patients with direct inguinal hernia, 65 patients with indirect inguinal hernia and only 13 with pantaloon hernia.

\section{Methods}

\subsection{Anesthesia}

General endotracheal anesthesia is used.

\subsection{The Technique}

A pneumoperitoneum is created using a Verres needle and an intra-abdominal pressure of $15 \mathrm{~mm} \mathrm{Hg}$ is maintained. The $10 \mathrm{~mm}$ trocar in infra-umbilical position is inserted. The telescope is then inserted and the intraabdominal cavity explored. Two $5 \mathrm{~mm}$ trocars are inserted lateral to each rectus muscle, at the same level as the umbilical trocar.

\subsubsection{Step 1: Creating the Peritoneal Flap}

The repair is initiated. The laparoscope is pointed toward the afflicted inguinal canal. The peritoneal defect or hernia is identified. The other inguinal canal is inspected. If an asymptomatic hernia sac is found on the other side, it is excluded from the study. The Lateral Umbilical Ligament is located as well as the Inferior Epigastric Artery and Vein. A peritoneal incision is made using the EndoShear*instrument connected to an electocautery source. The incision is extended from the lateral aspect of the inguinal region to the Lateral Umbilical Ligament as high as possible to maximize the exposure of the region.

\subsubsection{Step 2: Exposing the Inguinal Structures}

Cooper's Ligament is exposed as well as the Inferior Epigastric Vessels and the Spermatic Cord. It is essential to expose the uncovered abdominal wall meticulously (without peritoneum) and remove all fatty layers.

\subsubsection{Step 3: Dissecting the Hernia Sac}

The inguinal hernia sac should be dissected carefully from the Spermatic Cord. Particular care should be taken not to dissect lateral and inferior to Cooper's ligament, as the Iliac Artery and Vein will enter the femoral canal at this site.

\subsubsection{Step 4: Inserting and Anchoring the Mesh}

To 60 patients the mesh was stapled against the posterior surface of the anterior abdominal wall using automatic gun stapler, for the other 60 patients before inserting the mesh, its middle bilaterally is anchored to two prolene threads that are tied with the knots towards the back (Figures 1 and 2), to be facing the abdominal wall when it is pulled using the port closure device, which is introduced obliquely twice, first just medial to the anterior iliac spine, second lateral to midline (Figure 3), pulled (Figure 4) then tied in place. We used $8 \times 12 \mathrm{~cm}$ mesh to cover the myopectineal orifice applied onlay on cord structures.

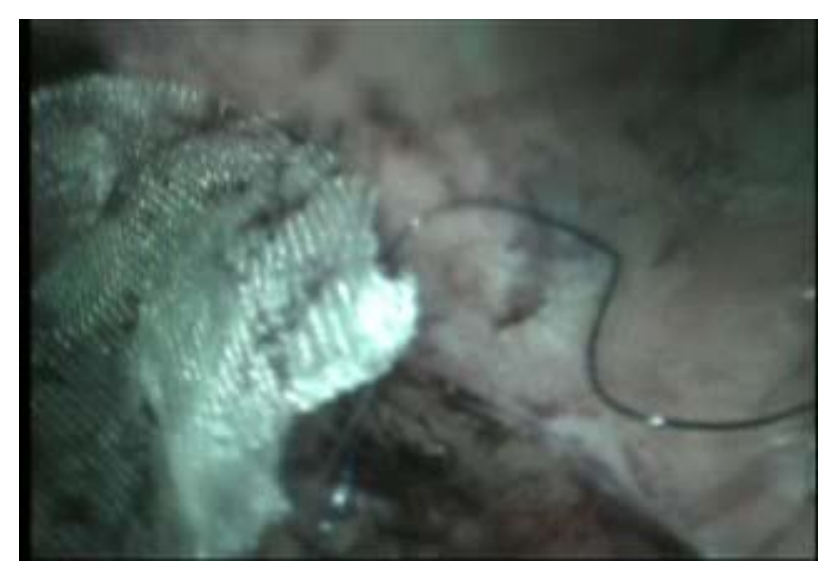

Figure 1. Lateral thread.

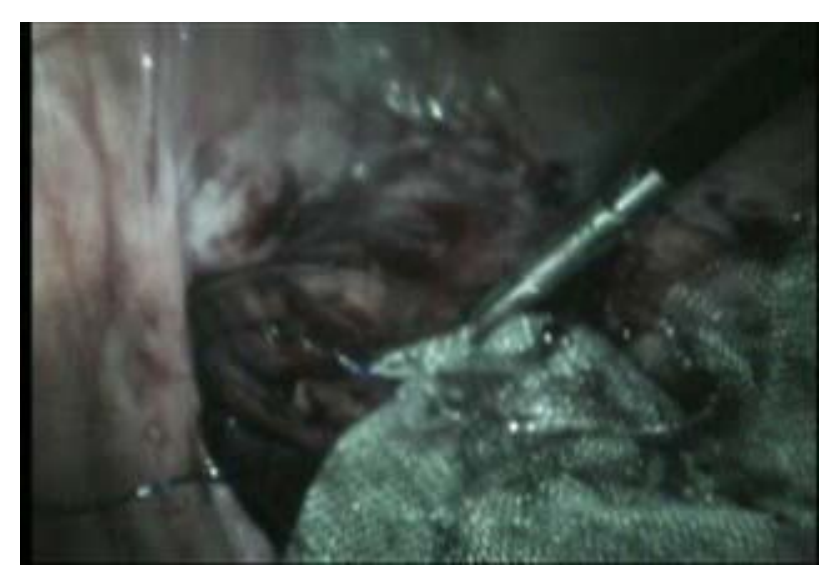

Figure 2. Medial thread.

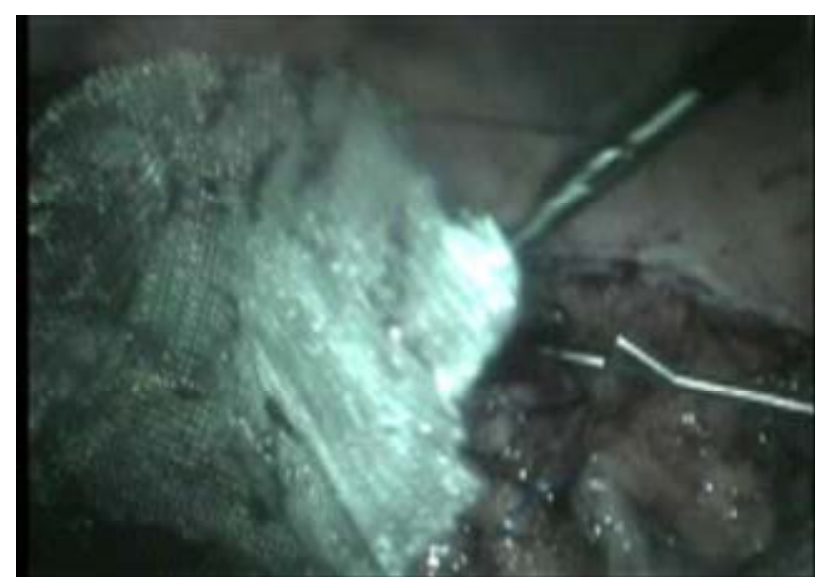

Figure 3. Port closure device opened. 


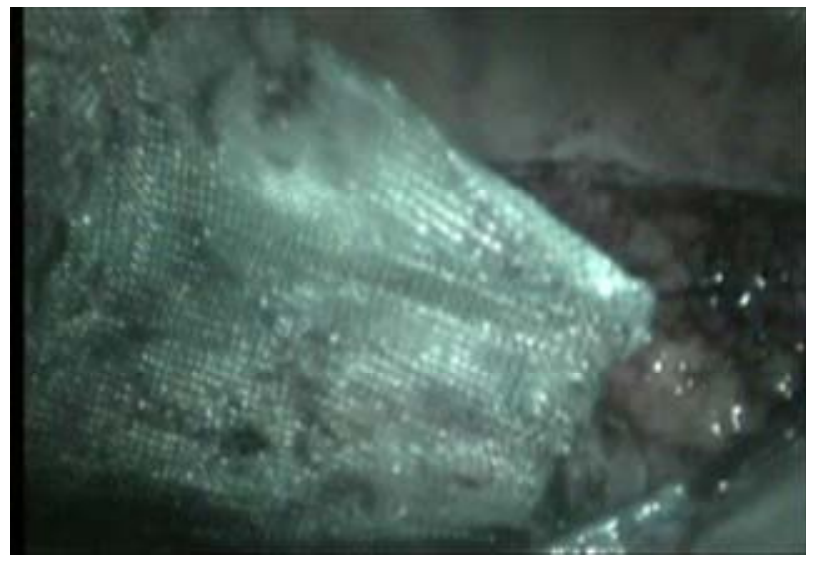

Figure 4. Pulling the lateral thread.

\subsubsection{Step 5: Closing the Peritoneum}

It is closed while reducing the pressure with vicryl 2/0.

For post operative pain relief, injection diclofenac sodium $75 \mathrm{mg}$ i.m. will be given post-operatively in the recovery room to all patients. Pain will be recorded at 1 , 6, 24 hours after operation, at the time of discharge on a Visual Analogue Scale (VAS) with end points labeled as no pain and worst possible pain on a scale of 10. (Visual analogue scale No pain Worst possible pain 0123456 $78910)$.

\section{Results}

All these patients underwent TAPP repairs. There were no visceral and vascular injuries with zero conversions to open surgery. The time of surgery ranged from 35 minutes to 70 minutes from the first incision until the last suture in the external fixation group of patients, on the stapled mesh group of patients ranged from 30 - 60 minutes. We followed patients until December 2012, so it ranged from 4 months until 51 months. During this period we did not encounter any recurrences.

Regarding pain no single patient required extra analgesic until discharge from the hospital except two patients in the stapled mesh group of patients who complaint of groin pain and was in need for more analgesics. The port sizes are reduced in external fixation group of patients with considerable reduction in the cost. The outlet puncture with the prolene thread showed no infection, no reaction at all until it is pulled and cut.

\section{Discussion}

Laparoscopic hernia repair was first described by Ger in 1990, who placed a simple mesh plug in the defect [12]. Laparoscopic inguinal hernia repair (LIHR) has comparable results to open hernia repair (OHR). Many studies have shown that LIHR gives similar results in terms of recurrence as OHR but with the added advantage of reduced post operative, pain, wound infection and early return to activity [13]. Several laparoscopic procedures have successfully passed the stage of feasibility assessment and are currently under scrutiny with regard to indications. Laparoscopic repair of inguinal hernia is a typical example of such investigations [14]. Mechanisms of recurrence may be related to technical difficulties and the use of inadequate mesh size and positioning [15]. A model that takes into account the additional equipment cost and time cost related to laparoscopic surgery, as patients return to work earlier. Unfortunately, this analysis showed that an early return to work does not offset the additional costs associated with laparoscopic hernia repair [16]. Reliable laparoscopic fixation of meshes prior to their fibrous incorporation is intended to minimize recurrences following transabdominal preperitoneal hernia (TAPP) [17]. It is not necessary to secure the mesh during laparoscopic TAPP inguinal hernia repair from the interior and fix it only to the exterior [18]. By all criteria of success-recurrence, recovery, long term symptoms and economics-laparoscopic inguinal hernia repair in the way we are doing is the winner. The end result on both groups nearly the same except that the operative time is longer in the external fixation group of patients. Two patients in the stapled mesh group of patients got groin pain with no such pain in external fixation of the mesh favoring the use of external fixation In external fixation group of patients we omitted the use of the disposable $12 \mathrm{~mm}$ trocar and the use of any hernia tucker which are the most expensive parts in the cost of TAPP hernia repair. Olmi et al. [19] stated that their experience demonstrates that fibrin glue (Tissocol) is an effective method for mesh fixation during TAPP, yet our method has the advantage of being fixed well. Kapiris et $a l$. [20] stated that TAPP repair is a technically demanding laparoscopic technique, but once mastered, is safe and effective with a high degree of patient satisfaction. Stapling the mesh is not necessary in most cases, thus resulting in a remarkably low cost. Again external fixation of the mesh is superior as it is associated with fixation and at the same time low cost. The issue of mesh fixation in laparoscopic repair of inguinal hernia repairs remains unsolved. The need for fixing the mesh arises from the fear of increasing recurrence rates. However, specific complications have emerged as a result of mesh fixation and in our study we got two patients with post TAPP groin pain. Avoid stapling of the mesh helps in decreasing complications and operative costs without affecting recurrence rates.

\section{Conclusion}

It is not necessary to secure the mesh during laparoscopic TAPP inguinal hernia repair from the interior and it is fixed only to the exterior allowing a reduction in size of the ports and considerable reduction in cost with less post 
TAPP pain with mild increase in operative time.

\section{REFERENCES}

[1] M. L. Wall, T. H. Cherian and J. C. Lotz, "Laparoscopic Hernia Repair-The Best Option?” Acta Chirurgica Belgica, Vol. 108, No. 2, 2008, pp. 186-191.

[2] A. Fingerhut, B. Millat, N. Veyrie, E. Chouillard and C. Dziri, “Inguinal Hernia Repair-Update 2006,” In: E. A. M. Neugebauer, S. Sauerland, A. Fingerhut, B. Millat and G. Buess, EAES Guidelines for Endoscopic Surgery, Springer, Berlin Heidelberg, 2006, pp. 297-309. http://dx.doi.org/10.1007/978-3-540-32784-4_14

[3] J. Wellwood, M. J. Sculpher and D. Stoker, "Randomised Controlled Trial of Laparoscopic versus Open Hernia Repair for Inguinal Hernia: Outcome and Cost,” British Medical Journal, Vol. 317, 1998, pp. 103-110. http://dx.doi.org/10.1136/bmj.317.7151.103

[4] D. M. Wright, A. Kennedy and J. N. Baxter, "Early Outcome after Open versus Extraperitoneal Endoscopic Tension-Free Hernioplasty,” Surgery, Vol. 119, No. 5, 1996, pp. 552-527.

http://dx.doi.org/10.1016/S0039-6060(96)80266-2

[5] K. W. Millikan, M. L. Kosik and A. Doolas, “A Prospective Comparison of Transabdominal Peritoneal Laparoscopic Hernia Repair versus Traditional Open Hernia Repair in a University Setting," Surgical Laparoscopy \& Endoscopy, Vol. 4, 1994, pp. 247-253.

[6] P. Kiruparan and S. H. Pettit, "Prospective Audit of 200 Patients Undergoing Laparoscopic Inguinal Hernia Repair with Followup from 1 to 4 Years," Journal of the Royal College of Surgeons of Edinburgh, Vol. 43, No. 1, 1998, pp. 13-16.

[7] D. C. Brooks, "A Prospective Comparison of Laparoscopic and Tension-Free Open Herniorraphy," Archives of Surgery, Vol. 129, No. 4, 1994, pp. 361-366. http://dx.doi.org/10.1001/archsurg.1994.01420280031004

[8] A. S. Seid and E. Amos, "Entrapment Neuropathy in Laparoscopic Herniorraphy," Surgical Endoscopy, Vol. 8, No. 9, 1994, pp. 1050-1053. http://dx.doi.org/10.1007/BF00705717

[9] C. G. Schmedt, S. Sauerland and R. Bittner, "Comparison of Endoscopic Procedures vs Lichtenstein and Other Open Mesh Techniques for Inguinal Hernia Repair," Surgical Endoscopy, Vol. 19, No. 2, 2005, pp. 188-199. http://dx.doi.org/10.1007/s00464-004-9126-0

[10] K. McCormack, B. Wake, J. Perez, C. Fraser, J. Cook and E. Mcintosh, "Laparoscopic Surgery for Inguinal Hernia Repair: Systemic Review of Effectiveness and Economic Evaluation,” Health Technology Assessment, Vol. 9, No. 14, 2005, pp. 1-203.
[11] P. Ridings and D. S. Evans, "The Transabdominal PrePeritoneal (TAPP) Inguinal Hernia Repair: A Trip along the Learning Curve," Journal of the Royal College of Surgeons of Edinburgh, Vol. 45, No. 1, 2000, pp. 29-32.

[12] E. A. M. Neugebauer, H. Troidi and C. K. Kum, "The EAES Clinical Practice Guidelines on Laparoscopic Cholecystectomy, Appendectomy and Hernia Repair,” In: E. A. M. Neugebauer, S. Sauerland, A. Fingerhut, B. Millat and G. Buess, Eds., EAES Guidelines for Endoscopic Surgery, Springer, Berlin Heidelberg, 2006, pp. 265-289. http://dx.doi.org/10.1007/978-3-540-32784-4_12

[13] J. Koniger, J. Redecke and M. Butters, “Chronic Pain after Hernia Repair: A Randomized Trial Comparing Shouldice, Lichtenstein and TAPP," Langenbeck's Archives of Surgery, Vol. 389, No. 5, 2004, pp. 361-365. http://dx.doi.org/10.1007/s00423-004-0496-5

[14] F. Tatulli, G. Chetta, A. Caputi, P. Mastrototaro and T. Ruggieri, “Laparoscopic Inguinal Hernia Repair: Audit of Our Experience with Laparoscopic Trans-Abdominal ProPeritoneal Repair (TAPP),” Chirurgia Italiana, Vol. 61, No. 1, 2009, pp. 47-53.

[15] A. Watson, P. Ziprin and S. Chadwick, "TAPP Repair for Inguinal Hernias-A New Composite Mesh Technique," Annals of The Royal College of Surgeons of England, Vol. 88, No. 7, 2006, p. 678.

[16] C. R. Voyles, B. J. Hamilton, W. D. Johnson and N. Kano, "Meta-Analysis of Laparoscopic Inguinal Hernia Trials Favors Open Hernia Repair with Preperitoneal Prosthesis," American Journal of Surgery, Vol. 184, 2002, pp. 6-10. http://dx.doi.org/10.1016/S0002-9610(02)00878-4

[17] R. Schwab, O. Schumacher, K. Junge, M. Binnebosel, U. Klinge and H. F. Becker, "Biomechanical Analyses of Mesh Fixation in TAPP and TEP Hernia Repair,” Surgical Endoscopy, Vol. 22, No. 3, 2008, pp. 731-738. http://dx.doi.org/10.1007/s00464-007-9476-5

[18] M. S. Abdelhamid, “Transabdominal Pre-Peritoneal Inguinal Hernia Repair with External Fixation,” Hernia, Vol. 15, No. 2, 2011, pp. 185-188. http://dx.doi.org/10.1007/s10029-010-0766-9

[19] S. Olmi, L. Erba, A. Bertolini and A. Scaini, "Fibrin Glue for Mesh Fixation in Laparoscopic Transabdominal Preperitoneal (TAPP) Hernia Repair Indications, Technique and Outcomes,” Surgical Endoscopy, Vol. 20, No. 12, 2006, pp. 1846-1850. http://dx.doi.org/10.1007/s00464-005-0502-1

[20] S. Kapiris, T. Mavromatis, S. Andrikopoulus, C. Georgiades, D. Floros and G. Diamantoupolus, "Laparoscopic Transabdominal Preperitoneal Hernia Repair (TAPP): Stapling the Mesh Is Not Mandatory,” Journal of Laparoscopic \& Advanced Surgical Techniques, Vol. 19, No. 3, 2009, pp. 419-422. http://dx.doi.org/10.1089/lap.2008.0350 